\title{
Goodman, Jane E. - Berber Culture on World Stage, from Village to Video
}

\section{Stéphanie Pouessel}

\section{OpenEdition}

1 Journals

Édition électronique

URL : http://journals.openedition.org/etudesafricaines/14096

DOI : 10.4000/etudesafricaines.14096

ISSN : 1777-5353

Éditeur

Éditions de l'EHESS

\section{Édition imprimée}

Date de publication : 8 décembre 2009

Pagination : 1018-1022

ISBN : 978-2-7132-2209-2

ISSN : 0008-0055

\section{Référence électronique}

Stéphanie Pouessel, «Goodman, Jane E. - Berber Culture on World Stage, from Village to Video », Cahiers d'études africaines [En ligne], 196 | 2009, mis en ligne le 08 décembre 2009, consulté le 21 septembre 2020. URL : http://journals.openedition.org/etudesafricaines/14096 ; DOI : https://doi.org/10.4000/ etudesafricaines.14096

Ce document a été généré automatiquement le 21 septembre 2020.

(c) Cahiers d'Études africaines 


\title{
Goodman, Jane E. - Berber Culture on World Stage, from Village to Video
}

\author{
Stéphanie Pouessel
}

\section{RÉFÉRENCE}

GoodmAN, Jane E. - Berber Culture on World Stage, from Village to Video. Bloomington, Indiana University Press, 2005, 239 p., biblio.

1 Comment est-ce que la culture voyage $^{1}$ ? Difficile de trouver question plus contemporaine. Jane E. Goodman nous invite ici à un voyage, du village kabyle à sa représentation dans la grande salle de spectacle de l'olympia à Paris. Comment un tel passage a-t-il pu être possible ? Plus précisément, quelles sont les instances, culturelles, politiques et scientifiques via l'anthropologie, qui ont conduit à la mise en scène de la culture kabyle dite traditionnelle?

2 Aujourd'hui professeur d'anthropologie au Département communication et culture de l'Indiana University de Boston, Jane E. Goodman a débuté en 1992 ce travail de recherche ethnographique qui l'implique directement. Elle a été accueillie au sein de familles kabyles, en Algérie et en France, tour à tour chanteuse dans la chorale de femmes kabyles ou adhérente d'une association de villageois émigrés à Paris. De plus, sa maîtrise de la langue kabyle lui permet de saisir les écarts de traduction entre ici et là-bas, tant linguistiques que culturels, qui définissent la culture berbère contemporaine.

3 Pour retracer ces réseaux d'« internationalisation» de la culture kabyle, l'ouvrage navigue entre la Kabylie et la France mais ailleurs aussi : c'est à Boston, en 1980, que la Kabylie a «atteint » l'auteure, quand elle a écouté pour la première fois la fameuse chanson du chanteur kabyle Idir «A Vava Inouva », à l'époque l'une des mélodies les plus populaires de la bande FM parisienne.

4 Comment la culture kabyle est-elle arrivée jusqu'à elle? En partant d'éléments «traditionnels », comme le mariage, la maison ou l'assemblée villageoise, elle souhaite 
comprendre l'actuelle revivification de la culture berbère. Poésie kabyle, proverbes, légendes sont aussi réinvestis comme source de culture et contribuent à la création de l'identité berbère contemporaine. Ce sont les bran-chements ${ }^{2}$ que Jane E. Goodman se propose d'appréhender.

5 Ce n'est donc pas tant la culture kabyle qui motive ses recherches mais bien plutôt sa mise en scène contemporaine, sa représentation. Plus loin que cela, c'est sa propre présence qui questionne la chercheuse : comment une Américaine en vient à filmer une chorale dans un petit village kabyle? «An American woman has come to view their culture. We are all part of the day's documentation. All of us are on stage » (p.1). Son point de départ interroge donc son propre questionnement: Pourquoi cet intérêt occidental aux « cultures du monde ", aux traditions ? Un point de départ réflexif fidèle à l'ensemble de l'ouvrage au cours duquel la chercheuse s'inclut constamment dans le texte ( I I felt like a spy »). Elle est avec les gens qu'elle étudie, ne l'omet jamais à la manière dont elle nous présente les hôtes qui l'ont tour à tour accueillie.

6 Comment l'identité culturelle berbère s'est-elle petit à petit constituée à travers et en direction de la scène mondiale? Quelles voies ont conduit les Kabyles à entrer dans la «modernité»? Quelles productions culturelles y ont contribué et surtout quelles conditions ont permis cette entrée?

7 Ce sont en effet d'abord des rencontres dépassant largement les frontières algériennes ("extralocal formations") qui ont formé la culture berbère kabyle contemporaine moderne. Grâce à cette focalisation exogène, l'auteure se départit d'une analyse qui réduirait la renaissance berbère à un projet néocolonial et l'inscrit dans les réseaux globaux actuels. La constitution indéniable de réseaux d'échanges, de branchements, donne en effet elle seule sens à l'identité berbère d'aujourd'hui. Dans les pas de JeanLoup Amselle et contre tout culturalisme, la chercheuse n'identifie une singularité, si kabyle soit-elle, que dans sa relation avec l'autre.

8 Dans l'exigence de cette posture relationnelle, Goodman, dont le premier terrain en 1992 s'oriente vers la nouvelle chanson kabyle, se trouve de suite face à des interconnections qu'elle s'engage alors à démêler. La nouvelle chanson berbère émerge dans les années 1970, en totale connexion avec les films ethnographiques, la théorie postcoloniale, l'idéologie panafricaine et le folklore occidental. La nouvelle chanson kabyle serait le médiateur-clé à travers lequel les notions de l'identité amazighe ont été diffusées.

9 Le type de sujet décentré et diasporique que propose l'auteure mène à une remise en cause du «terrain » traditionnel de l'ethnographie. Dans le sillon de G. E. Marcus qui propose un terrain "multisite $»^{3}$, elle le définit non plus comme un lieu au sens géographique mais comme «a problematic that emerges at the intersection of contemporary ideologies, historically shaped discursive formations, and situated discourse and performance practices » (p. 20).

10 Cette volonté de vouloir comprendre le lien entre culture vécue, non revendiquée, et culture berbère telle qu'elle est mise en avant en France, amène l'auteure à retracer les réseaux de diffusion de "la culture » kabyle : le courant "world music », l'université et la diaspora kabyle.

11 Des événements clés ont participé à la constitution d'une identité amazighe contemporaine : ce fut le cas de Tafust imazighen. En retraçant, heure par heure, les événements du " printemps berbère ", dont l'étincelle ayant mis le feu aux poudres (la 
censure de la conférence de Mouloud Mammeri), l'auteure soutient que avril 1980 a été un tournant dans le développement de la conscience berbère. La spécificité de cette répression (une parmi d'autres) réside dans la rapidité de sa médiatisation par plusieurs réseaux internationaux: l'université (lieu crucial de formulation et de dissémination du discours sur l'identité amazighe), les élites à Paris (l'Académie berbère a fait de Paris le siège de la formulation du discours sur l'identité amazighe dans les années 1960-1970; son journal, qui diffuse les signes tifinagh, circule en Kabylie illégalement créant un réseau décentralisé) et les organisations des droits de l'Homme via la presse internationale.

D'autres points de référence restent cruciaux dans la constitution d'une identité berbère comme la chanson "A Vava Inouva ». Comment cette chanson d'Idir a-t-elle constitué une des premières pièces d'un «héritage collectif nouveau»? Premier hit algérien en France, et diffusée à la station radio kabyle, cette chanson a eu sur les Kabyles un effet «electrifying» (p. 49). Perçue comme le "regard intérieur», un mode indigène de connaissance, elle constitue en fait déjà un regard décalé puisqu'elle est construite à travers des événements et des entités géopolitiques distants. Le festival pan-africain de 1969 à Alger (à l'initiative de l'Organisation de l'Union africaine) constitue un des branchements puisqu'il a marqué Ben Mohamed, le parolier de «A Vava Inouva » («It was there that I began to grasp what it meant to belong to a culture »[p. 53]). Dans un rapport triangulaire avec la France, à travers la fierté de la communauté nord africaine en France, le succès hexagonal du hit produit un effetfeedback en Algérie.

13 Censé représenter un regard presque ethnographique, c'est-à-dire de l'intérieur, ce succès musical illustre davantage une "vernacular modernity » (p. 52) : c'est le passage par des canaux «extérieurs" qui contribue à l'objectivation de la culture. L'ethnographie vient ici comme un miroir permettant l'objectivation de la culture et contribue ainsi à la prise de conscience, au regard sur soi.

Enfin, le troisième référent central de la « kabylité » prend appui sur le village berbère. Véritablement pris à parti dans la construction contemporaine de la culture kabyle, la focalisation sur cet espace «traditionnel » tranche avec la volonté de modernisation de ses habitants. En remontant aux prémisses de l'idéalisation du village, les administrateurs coloniaux français ont retrouvé dans le village kabyle « a romanticized reflection of Europe's own rural past, while fashioning the Kabyles as a peasantry of small landowners who would assimilate easily into the colonial state» (p. 71). La sociologie, de son côté, contribue à l'élaboration de ce mythe, à travers Pierre Bourdieu et sa fameuse idéalisation de la Kabylie précoloniale qui, au côté d'Abdelmalek Sayad, produisit une sociologie essentialiste du village. Bourdieu perçoit l'arrivée de l'armée française comme une rupture sans précédent, de « pathological acceleration of cultural change» (p.72), alors que le village est tout sauf une entité originelle stable et stagnante mais bien en mouvement, "an organization of circulation» (p. 72). Un mouvement illustré aujourd'hui par ses forts taux d'émigration: dans le village où séjourna Goodman durant sa recherche, deux tiers de ses habitants vivaient à Paris ! Ce qui amène l'auteure à parler de "trans-Mediterranean village » (p. 73). L'immigration, mais aussi le "développement", lui offrent deux axes de désessentialisation qui permettent ainsi de décloisonner le village kabyle.

15 La maison kabyle jouit du prestige de sa description bourdieusienne : «A microcosm organized by the same oppositions and the same homologies which order the whole 
universe » (p. 77). Pour Goodman - qui reprend les travaux de Bourdieu, mais pour les dépasser - la maison est en fait centrale car presque toute l'année vide, et marque pendant l'absence de l'émigré sa présence au sein du village. Cette présence-absence lui permet de maintenir la fiction de son retour (A. Sayad) ${ }^{4}$.

Grâce à une description minutieuse (elle dénombre quarante voitures au sein du village dans lequel elle réside) et surtout contemporaine (le contexte de forte émigration, la connexion du village par les ondes), Goodman œuvre à la déconstruction du «village kabyle». Loin de la conception statique de Bourdieu, ou celle plus antérieure des administrateurs coloniaux (Masqueray, Hanoteau, etc.), le village est d'abord décrit comme un carrefour.

est de même pour d'autres " éléments traditionnels » telle la tajma’at (assemblée). Relatée par les travaux ethnographiques comme une «démocratie primitive » et séculaire, l'auteure étudie sa reproduction à Paris, donc forcément ses transformations. Goodman dévoile également la dialectique caractérisant le mariage - institution pensée comme statique-, celle de la présence-absence faisant vivre les villages, particulièrement visible à travers la célébration des mariages qui se déroulent l'été, seule période où le village retrouve sa chair! Le mariage et l'assemblée changent constamment de forme, ce qui permet à l'auteure d'introduire la notion de mouvement: «The village in motion » (p. 93).

Ladite tradition orale joue aussi pour beaucoup dans l'érection de la culture kabyle contemporaine : poèmes, proverbes et histoires sont collectés depuis la conquête de la Kabylie jusqu'à aujourd'hui. Dépositaires de l'histoire sociale, ils deviennent de véritables actes politiques. Censés transposer l'esprit, l'âme berbère, le sens est en fait réinterprété selon le contexte actuel. Cette tradition orale contemporaine passe par la perception d'entités culturellement distinctes entre poésie arabe et poésie berbère. L'auteure nous rappelle pourtant que ce couple conflictuel arabe/berbère n'a pu exister qu'avec un tiers que fut le regard français.

19 La diffusion de l'identité berbère via la production poétique passe également par la production "endogène» et les paradoxes qu'elle porte en elle: Comment Jean Amrouche, en chantant les poèmes de sa mère censés refléter « la » culture kabyle dans son intégrité, exprimait en fait son hybridité de personnage berbère, tunisien, chrétien, écrivain en français. Issus de l'élite africaine éduquée en français, Jean Amrouche et sa sœur Taos ont inscrit les poèmes berbères dans les catégories européennes de littérature et de folklore.

20 Comment une chanson villageoise peut-elle passer à un registre universel, celui d'une nouvelle production culturelle porteuse de l'identité kabyle moderne, et s'inscrire ainsi sur la scène mondiale en tant que « world music »?

21 Jane E. Goodman procède à une analyse de textes (recueils de poésie) minutieuse : notes de bas de page, introduction, traduction, différence entre les versions françaises et kabyles. À travers Idir et son parolier Ben Mohamed, vivant tous deux à Paris, Goodman remonte le fil de la création artistique en réinvestissant ses sources: les textes des poèmes traditionnels. Elle se penche en effet sur la manière dont Idir et Ben ont retravaillé des chansons villageoises (textes anciens), les transformant ainsi en véhicules potentiels de la culture kabyle moderne. Pour ce faire, elle analyse le niveau d'altération des anciens textes (suppressions de certains éléments : religion, marabout, référence aux Turcs, aux Français), les contextes inédits (nouveaux instruments, un chanteur masculin solo, airs nationaux et internationaux, etc.) et l'attribution de 
nouveaux sens. La volonté des auteurs est double: rendre ces textes profondément berbères et intelligibles par tous. L'étude de la genèse des textes du chanteur permet de repérer le passage d'un registre « local », « traditionnel » (une famille, un mariage) à un registre universel, à un langage dont le sens devient compréhensible par tous : « Kabyle poetry collection has been a means of configuring Berber culture in relation to metropolitan, nationalist, and postcolonial ideas and ideologies » (p. 118).

En partant du cas précis de la chanson kabyle, Goodman apporte des éléments sur le processus à l'œuvre dans la globalisation des cultures, donc dans toutes les renaissances culturelles contemporaines. Sa réussite est d'avoir su dévoiler les coulisses d'un champ identitaire, sans tomber dans une quelconque posture dénonciatrice trop souvent génératrice d'«un désenchantement relativiste peu soucieux de l'institutionnalisation du social $»^{5}$.

La boucle se referme sur la représentation d'un village traditionnel berbère à l'olympia. L'ouvrage, telle une enquête, a débuté par le tournage sur une chorale d'enfants dans un village kabyle et s'achève sur le film d'une représentation berbère à Paris. Goodman a tenté d'élucider le passage de l'un à l'autre, de la tradition (le village) à sa mise en scène (l'olympia). Cette vidéo transporte avec elle des images idéalisées du pays générant une vision unifiée d'une identité collective. Celle-ci nie sa propre localisation et les conditions de sa création qui sont celles d'un espace transméditerranéen profondément hétérogène.

En retraçant une partie de l'itinéraire des discours qui a indubitablement inscrit les Kabyles sur la scène mondiale, Goodman nous offre une contribution majeure à l'anthropologie de la globalisation culturelle : en s'arrêtant sur des éléments minutieux issus du référent kabyle, elle dévoile lebackground des productions culturelles (textes ou événements), c'est-à-dire leur historicité et leur hybridité.

\section{NOTES}

1. Greg URBAN, Metaculture. How Culture Moves through the World, Minneapolis, University of Minnesota Press, 2001.

2. Jean-Loup AmSELLE, Branchements. Anthropologie de l'universalité des cultures, Paris, Flammarion, 2001.

3. E. MARCUS, «Ethnography in/of the World System: the Emergence of Multi-Sited Ethnography ", in E. MARCUS, Ethnography through Thick and Thin, Princeton, Princeton University Press, 1998, pp. 79-104.

4. Abdelmalek SAYAD, La Double absence. Des illusions de l'émigré aux souffrances de l'immigré, Paris, Éditions du Seuil, 1999.

5. Martina AVANZA \& Gilles LAFERTÉ, «Dépasser la "construction des identités"? Identification, image sociale, appartenance », Genèses, 61, 2005, pp. 154-167. 